\title{
Bacterial tracking of motile algae assisted by algal cell's vorticity field
}

\author{
J. T. Locsei and T. J. Pedley
}

November 4, 2018

\begin{abstract}
A short version of the title for running headings is 'Bacterial tracking of motile algae'.

Both authors are based at the

Department of Applied Mathematics and Theoretical Physics

Centre for Mathematical Sciences

University of Cambridge

Cambridge CB3 0WA

United Kingdom

J. T. Locsei is the corresponding author, and may be contacted at j.t.locsei@damtp.cam.ac.uk or $+44(0) 7849829002$
\end{abstract}




\begin{abstract}
Previously published experimental work by other authors has shown that certain motile marine bacteria are able to track free swimming algae by executing a zigzag path and steering toward the algae at each turn. Here, we propose that the apparent steering behaviour could be a hydrodynamic effect, whereby an algal cell's vorticity and strain-rate fields rotate a pursuing bacterial cell in the appropriate direction. Using simplified models for the bacterial and algal cells, we numerically compute the trajectory of a bacterial cell and demonstrate the plausibility of this hypothesis.
\end{abstract}




\section{Introduction}

The experiments of Barbara and Mitchell [3] demonstrate that certain species of marine bacteria are able to track motile algae by a combination of reversing direction and steering, presumably to make use of nutrients exuded by the algae. Their results raise a number of interesting questions about how the bacteria are able to perform this feat, such as (i) how do they steer, and (ii) how do they decide when to reverse. In this paper we shall address the first of these questions: steering. We hypothesise that a bacterial cell exploits the vorticity and strainrate fields generated by an algal cell in order to steer. Before presenting our calculations, we give a brief overview of bacterial chemotaxis.

Bacteria play an important role in marine ecosystems. Field studies indicate that approximately half of oceanic primary production (carbon fixed by phytoplankton) is channelled via bacteria into the microbial loop of the pelagic food web (2] and references therein). This remarkable rate of bacteria-mediated transformation of organic matter is facilitated by bacterial motility. It is estimated that 20 to $70 \%$ of planktonic bacteria are motile [12, 9]. Motility allows marine bacteria to locate sources of nutrients, for instance by colonising falling sinking aggregates ('marine snow') [18], or tracking motile marine algae [3] which leak organic matter [25].

The most studied and well-understood motile bacterium is Escherichia coli, an enteric bacterium, but marine bacteria differ from $E$. coli in several important ways. First, many species of marine bacteria swim much faster than $E$. coli, with maximum speeds up to $400 \mu \mathrm{m} \mathrm{s}^{-1}$ [24, 29], compared to typical speeds of $30 \mu \mathrm{m} \mathrm{s}^{-1}$ for E. coli [7]. Second, marine bacteria are able to respond to changes in chemoattractant concentrations within a fraction of a second, as evidenced by their ability to form tight clusters [27], whereas $E$ coli respond to changes in chemoattractant concentrations over the course of several seconds [32]. Finally, whereas $E$. coli moves in a run-and-tumble motion [5], many strains of marine bacteria move in a run-and-reverse motion 22, 27. Mechanistically, this is explained by the fact that $E$. coli has several flagella which may either bundle together to propel the cell in a straight 'run', or fly apart to make the cell 'tumble' [35], whereas many marine bacteria have only a single polar flagellum which may spin either one way or the other for a 'run' or 'reverse'. (For a review of other types of bacterial motions, see [28].) It has been suggested that run-and-reverse motion favoured by marine bacteria allows them to perform chemotaxis (swim toward a food source) more effectively in a turbulent environment, compared to run-and-tumble motion [23]. Other theoretical studies indicate that run-and-reverse may be a favourable strategy when moving up a uniform chemoattractant gradient in a shear flow [21] or when an organism is capable of direct gradient detection 22].

It seems that the run-and-reverse motion of marine bacteria is also advantageous when following a moving target such as a motile algal cell. Barbara and Mitchell [3] reported that motile marine bacteria of the species Pseudoalteromonas haloplanktis and Shewanella putrefaciens were able to track motile marine algae of the species Pavlova lutheri by moving in zigzag paths and apparently steering, consecutively turning up to 12 times toward the algae (figure 1). However, $P$. haloplanktis and $S$. putrefaciens cells have a single polar flagellum [10, 14], and it is not clear how they could actively steer with such an apparatus. Here, we suggest that the apparent steering is in fact a passive hy- 


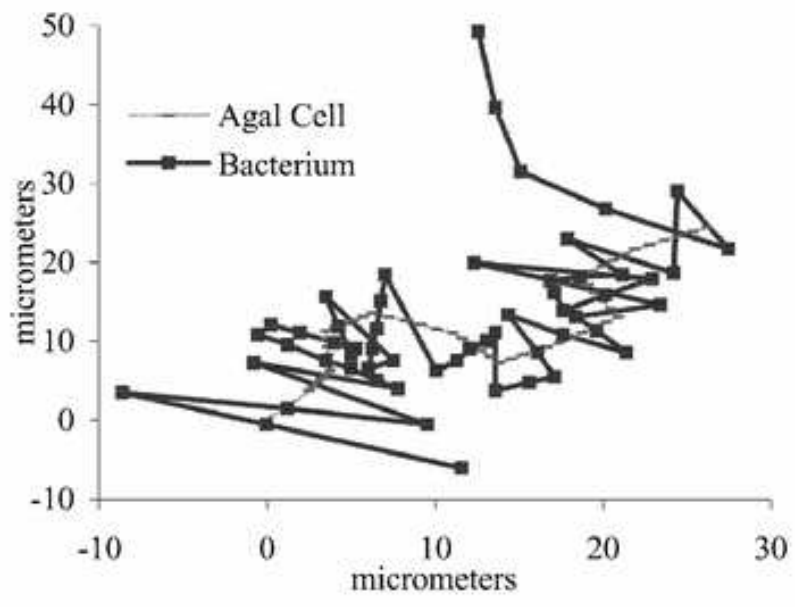

Figure 1: Observations of the paths of a $P$. lutheri algal cell being tracked by a $P$. haloplanktis bacterial cell (reproduced from figure 1a of [3], with permission). The algal cell track starts at the bottom-left of the figure. Each square represents a single data point; data was collected at 24 frames/sec.

drodynamic effect, whereby an algal cell's vorticity and strain rate fields rotate the pursuing bacterial cell in the appropriate direction. Sections 2.1 and 2.2 introduce simplified models for the bacterial and algal cells, based on what is known about them from experiments, and section 2.3 presents a scaling argument to show that the algal cell's vorticity field has the right order of magnitude to account for the observed bacterial steering. Section 2.4 describes a method for simulating bacterial tracking, and section 3 presents simulation results and investigates how parameters such as swimming speeds influence the success of tracking. Section 1 summarises the main findings and their biological relevance.

\section{Methods}

\subsection{Model algal cell}

The $P$. lutheri algal cells used in the experiments of [3] had diameters of approximately $6 \mu \mathrm{m}$ and swam at average speeds of $v_{\text {alg }} \approx 40 \mu \mathrm{m} \mathrm{s}^{-1}$. They changed direction about three times per second, and bacterial cells were able to track them despite these turns, but we ignore this complication and let the model algal cell swim in a straight line. All algae of the order Pavlovales have approximately spherical cell bodies and two flagella of unequal length, the longer of which is covered in fine hairs [11]. The flagella lengths of P. lutheri have not been measured, but in the related species $P$. gyrans the length of the longer flagellum is about $10-20 \mu \mathrm{m}$, and in $P$. mesolychnon the length of the longer flagellum is about $15-20 \mu \mathrm{m}$. The details of the flagellar beat pattern of $P$. lutheri are not well characterised, but it is known that the longer flagellum is directed forward during swimming. Thus, a P. lutheri cell is a 'puller', since it pulls itself forward with its longer flagellum, as opposed to a 'pusher' such 


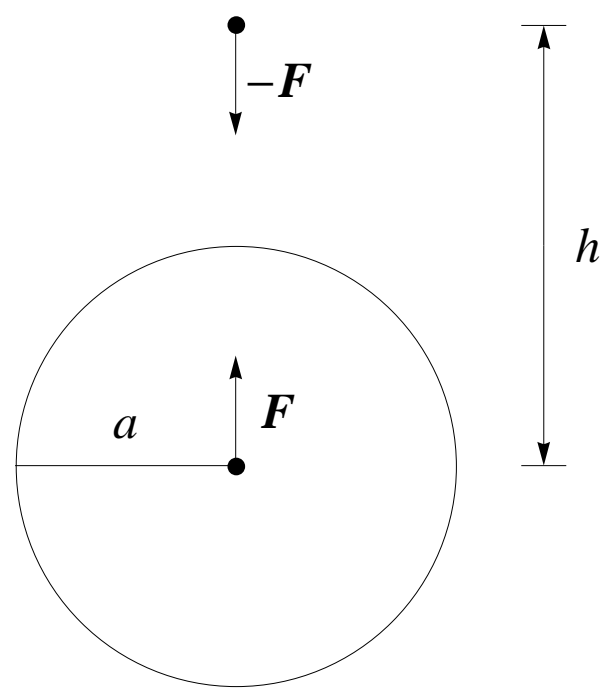

Figure 2: Simplified model of a $P$. lutheri algal cell, consisting of a sphere of radius $a$ acted upon by a force $\boldsymbol{F}$ and a Stokeslet of strength $-\boldsymbol{F}$ located a distance $h$ ahead of the sphere (the 'phantom flagellum'), such that the cell as a whole is force-free. In the orientation depicted here, the cell is swimming upward.

as Escherichia coli whose propulsive flagella bundle lies behind the cell as it swims.

In the absence of detailed knowledge of the flagellar beat pattern, we use a simplified model for a $P$. lutheri cell (figure 2). The cell body is treated as a sphere of radius $a=3 \mu \mathrm{m}$ acted upon by a force $\boldsymbol{F}$ in the swimming direction - this force is intended to model the tension at the base of the flagellum. The influence of the flagellum on the fluid is modelled as a point force $-\boldsymbol{F}$ acting on the fluid at a distance $h$ ahead of the cell body, with $h-a$ equal to approximately half the length of the longer flagellum. (This point force 'phantom' flagellum is analogous to that used in 13 for modelling motile bacteria.) We choose $h=10 \mu \mathrm{m}$, but our results are qualitatively unchanged for $h$ as low as $1 \mu \mathrm{m}$ and as high as $20 \mu \mathrm{m}$. Note the algal cell's velocity field in this model is constant in time, whereas in reality it is likely to vary periodically in time with the flagellar beat pattern, as well as vary over a longer time-scale as the algal cell changes its swimming speed.

The Reynolds number for the algal cell, based on the cell diameter, swimming speed and the viscosity and density of water, is $\operatorname{Re} \approx 10^{-4}$. Hence, the fluid flow around the cell is viscous, non-inertial, and governed by the Stokes equations, assuming that the fluid is Newtonian. Using the linearity of the Stokes equations, the velocity field around the algal cell is obtained by adding the solution for a translating sphere to the solution for a Stokeslet outside a stationary sphere [17]; the resulting expression is lengthy and is omitted here. Figure 3 shows the vorticity field around the model algal cell, and figure 1 shows the direction of maximum extension rate of the strain rate field of the model algal cell. 


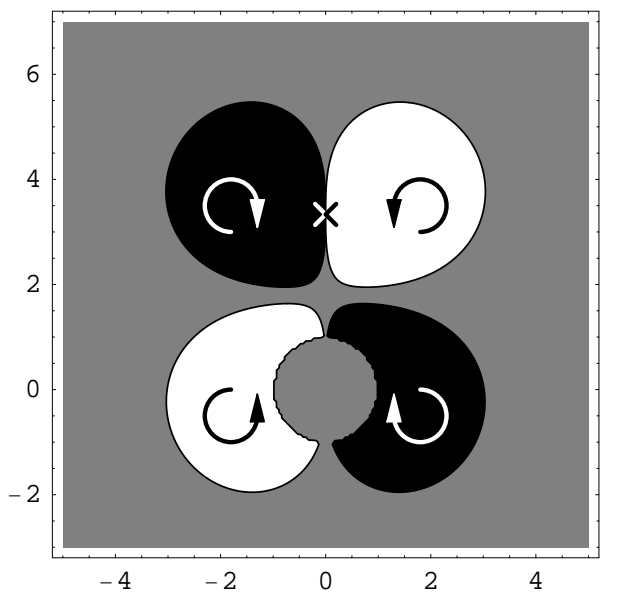

Figure 3: Cross section of the vorticity field around the model algal cell. Black and white regions are where the magnitude of the vorticity field exceeds $0.2 v_{\mathrm{alg}} / a$, and grey regions are where the magnitude of the vorticity field is less than $0.2 v_{\text {alg }} / a$. Arrows show the vorticity direction. The cell body is centered on the origin, and the phantom flagellum is marked by a cross. Scale is in units of the cell radius $a$.

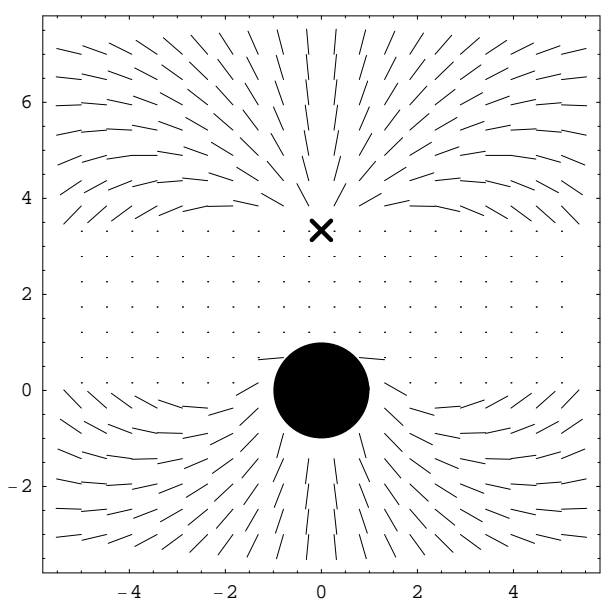

Figure 4: Direction of maximum extension rate of the strain rate field of the model algal cell. (An elongated body, such as a pursuing bacterium, will tend to align with the direction of maximum extension rate.) The cell body is shown as a black disk and the phantom flagellum is marked by a cross. Scale is in units of the cell radius $a$. In the blank regions, the direction of maximum extension rate is perpendicular to the page. 


\subsection{Model bacterial cell}

The two species of bacteria used in the experiments of [3], Pseudoalteromonas haloplanktis and Shewanella putrefaciens, swam at average speeds of $v_{\mathrm{bac}} \approx$ $200 \mu \mathrm{m} \mathrm{s}^{-1}$ while tracking algae. Cells from each species have a single, helical, polar flagellum 10, 14, which may rotate either counterclockwise or clockwise for a 'run' or 'reverse'. Barbara and Mitchell [3] did not report the size of the bacterial cells used in their study, but cells of all Pseudoalteromonas species are rod shaped with width 0.2 to $1.5 \mu \mathrm{m}$ and length 1.8 to $3 \mu \mathrm{m}$ [10], while those of the Shewanella species are rod shaped with width 0.4 to $0.7 \mu \mathrm{m}$ and length 2 to $3 \mu \mathrm{m} \mathrm{36}$. In each case the length of the flagellum is a few times that of the cell body. For the purpose of modelling we treat a swimming bacterial cell as a prolate spheroid of infinitesimal size which translates along its long axis and is rotated and advected by the velocity field of the algal cell. Ideally, one should incorporate the finite size of the bacterial cell and the influence of its velocity field on the algal cell, but to simplify the mathematics we neglect these complications.

Cells of E. coli, which are a similar size to P. Haloplanktis and S. putrefaciens, exhibit small, random changes in direction, and these have been attributed to rotational Brownian motion (i.e. thermal collisions with molecules in the surrounding fluid). Berg [- 1 estimated the the rotational diffusion coefficient due to Brownian rotation to be $D_{\mathrm{R}} \approx 0.062$ radians $^{2} \mathrm{~s}^{-1}$ for an $E$. coli cell swimming in a fluid of viscosity $2.7 \mathrm{cp}$ at $32^{\circ} \mathrm{C}$, and this is consistent with experimental observations [5]. However, this estimate was based on treating the $E$. coli cell as a sphere of diameter $2 \mu \mathrm{m}$, and when one takes into account the stabilising effect of the flagellar bundle, the theoretically predicted rotational diffusion coefficient is an order of magnitude smaller than the observed rotational diffusivity ( $\mathrm{H}$. Fu, personal communication), so it seems likely that the observed rotational diffusivity is in fact due to intrinsic "wobbly swimming' rather than true (thermal) Brownian rotation (H. Berg, personal communication). It is therefore unclear what value of $D_{\mathrm{R}}$ is appropriate for $P$. Haloplanktis and $S$. putrefaciens. In section 3 we shall simply use a default value of $D_{\mathrm{R}}=0.062$ radians $^{2} \mathrm{~s}^{-1}$, and demonstrate that the results have only a weak dependence on $D_{\mathrm{R}}$ anyway.

Given our assumptions, the translational velocity of the model bacterial cell is

$$
\dot{\boldsymbol{x}}=v_{\mathrm{bac}} \boldsymbol{p}+\boldsymbol{u}(\boldsymbol{x}, t)
$$

where $\boldsymbol{x}$ is the position vector of the bacterial cell, $v_{\mathrm{bac}}$ is the bacterial cell's swimming speed, $\boldsymbol{p}$ is a unit vector denoting the swimming direction of the bacterial cell, and $\boldsymbol{u}(\boldsymbol{x}, t)$ is the velocity field due to the algal cell at the position of the algal cell. We treat $v_{\text {bac }}$ as a constant, although in the experiments of [3] the bacterial cells often altered their speeds by a factor of 2 or more during tracking.

With the exception of reversals and collisions (discussed later), the rate of change of direction of the bacterial cell is

$$
\dot{\boldsymbol{p}}=\frac{1}{2} \boldsymbol{\omega} \times \boldsymbol{p}+\alpha_{0} \boldsymbol{p} \cdot \boldsymbol{E} \cdot(\boldsymbol{I}-\boldsymbol{p} \boldsymbol{p})+\boldsymbol{\xi} \times \boldsymbol{p} .
$$

The first two summands on the right hand side of (2) represent the deterministic rotation of the bacterial cell by the vorticity field $\boldsymbol{\omega}$ and strain-rate field $\boldsymbol{E}$ of 
the algal cell 20], and

$$
\alpha_{0}=\left(\eta^{2}-1\right) /\left(\eta^{2}+1\right)
$$

where $\eta$ is the ratio of the major to minor axis of the cell (slenderness ratio). We choose $\eta=10$ as a default value (suggested as a reasonable value by [21]), and we explore the effect of different values for $\eta$ in section 3. The last term on the right hand size of (2) represents rotational diffusivity, with $\boldsymbol{\xi}$ being a random, white-noise angular velocity with autocorrelation function

$$
\left\langle\xi_{i}\left(t_{1}\right) \xi_{j}\left(t_{2}\right)\right\rangle=D_{\mathrm{R}} \delta_{i j} \delta\left(t_{1}-t_{2}\right)
$$

An important ingredient of the model is a rule to decide when the bacterial cell reverses direction. The details of the chemotactic response of marine bacteria are yet to be characterised [27]. For instance, it is not known whether they perform temporal comparisons of chemoattractant concentrations like E. coli [32], whether they directly detect chemoattractant gradients, or whether they respond to absolute chemoattractant concentrations. Barbara and Mitchell [3] observed that during tracking a bacterial cell remained within a distance of about $6 \mu \mathrm{m}$ from the algal cell. Based on this, we choose the simplest rule for bacterial reversals, and make the model bacterial cell reverse direction whenever it reaches a 'reversal distance' $R \approx 6 \mu \mathrm{m}$ from the centre of the model algal cell.

One way that the bacterial cell might achieve this in reality is to reverse direction whenever the chemoattractant concentration drops below a certain threshold. The chemoattractant in the experiments is likely to have been dissolved oxygen released by the algal cells (J. G. Mitchell, personal communication), which has a diffusion coefficient of approximately $2000 \mu \mathrm{m}^{2} \mathrm{~s}^{-1}$ in water at room temperature. Given an algal cell swimming speed of $40 \mu \mathrm{m} \mathrm{s}^{-1}$ and an algal cell radius of $3 \mu \mathrm{m}$, the Péclet number is $\mathrm{Pe} \approx 0.06 \ll 1$. If the chemoattractant is a small organic molecule instead, then a commonly used diffusion coefficient is $1000 \mu \mathrm{m}^{2} \mathrm{~s}^{-1}$ (used for instance in [31, 15, 16, 23, 60), which gives $\mathrm{Pe} \approx 0.12 \ll 1$. In either case, diffusion dominates over advection and to leading order the distribution of chemoattractant concentration $c$ in the near vicinity of the algal cell is spherically symmetric with distribution

$$
c(r)=c_{0} a / r,
$$

where $c_{0}$ is the concentration on the surface of the algal cell and $r$ is distance from the centre of the algal cell. So, if the bacterial cell reverses direction when, say, $r=R=2 a$, the corresponding concentration threshold is $c_{0} / 2$.

The final ingredient for the model bacterial cell is a rule for what happens when it collides with the algal cell. In reality, there will be some complicated interaction involving a lubrication flow between the two cells, and potentially also an active response from one or both of the cells. Hydrodynamic effects are know to cause bacteria propelled by helical flagella to swim along curved paths when they are near a planar surface (\$ 8 and references therein), and a similar effect might occur in the interaction between the bacterial and algal cells. For the purpose of the model, however, we assume that when the bacterial cell collides with the algal cell, it simply glances off and its swimming direction changes so as to be parallel with the surface of the algal cell. Mathematically, the bacterial swimming direction $\boldsymbol{p}_{1}$ after collision is related to the swimming direction $\boldsymbol{p}_{0}$ before collision by

$$
p_{1}=\frac{u}{\sqrt{u \cdot u}}
$$




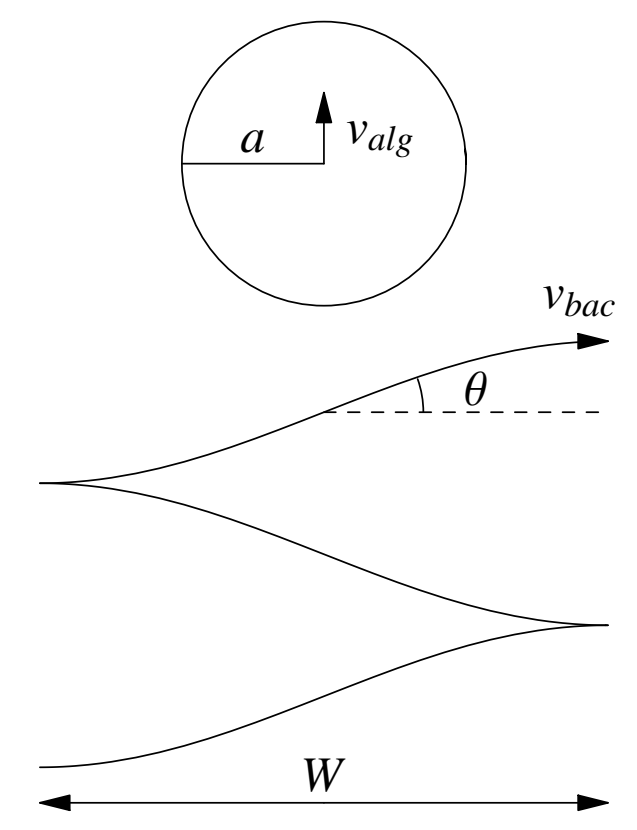

Figure 5: Idealised zigzag path of bacteria tracking algae.

where

$$
\boldsymbol{u}=(\boldsymbol{I}-\boldsymbol{n n}) \cdot \boldsymbol{p}_{0},
$$

$\boldsymbol{I}$ is the identity matrix, and $\boldsymbol{n}$ is the outward unit normal vector from the algal cell surface.

\subsection{Scaling argument}

Imagine a bacterial cell performing run-and-reverse motion in a side to side fashion (with respect to the algal cell's velocity) just behind a swimming algal cell. The algal cell's vorticity field (figure 3) rotates the bacterial cell such that instead of retracing the same path over and over, the bacterial cell traces out a curved zigzag path as depicted in figure 5, with a net motion in the same direction as the algal cell's velocity.

A simple scaling argument suggests that the algal cell's vorticity field alters the bacterial cell's path by the right order of magnitude for the bacterial cell to keep pace with the algal cell. If the bacterial cell is within a distance $R$ of the algal cell, then the magnitude of the vorticity field it experiences is $\omega \sim v_{\text {alg }} / R$, where ' $~$ ' means 'is of order'. Let $W$ be the width of the zigzag path followed by the bacterial cell, and let $\theta$ be the angle between the bacteria cell's swimming direction and the plane normal to the algal cell's swimming direction (see figure 5). The angle $\theta$ is approximately the angle through which the bacterial cell is rotated by the vorticity field as it swims the width of the zigzag, so $\theta \sim$ $\omega W / v_{\text {bac }} \sim\left(v_{\text {alg }} / v_{\text {bac }}\right) W / R \sim v_{\text {alg }} / v_{\text {bac }}$, since $W \sim R$. Let us assume that $v_{\text {bac }} \gg v_{\text {alg }}$, so that $\theta \ll 1$. Then, the component of the bacterial cell's velocity parallel to the algal cell's velocity is $v_{\mathrm{bac}} \sin \theta \sim v_{\mathrm{bac}} \theta \sim v_{\mathrm{alg}}$. Hence the algal cell's vorticity field has the right order of magnitude for the bacterial cell to 


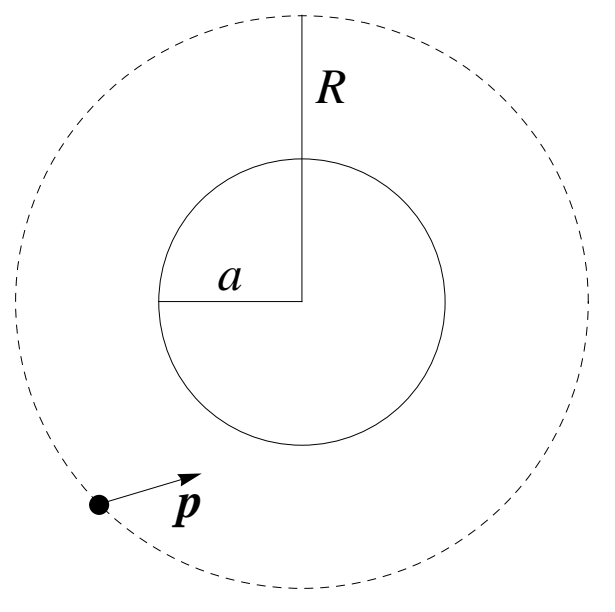

Figure 6: Example initial condition. Circle of radius $a$ denotes the algal cell. Bacterial cell initial position is random and lies a distance $R$ from the centre of the algal cell. Bacterial initial swimming direction $\boldsymbol{p}$ is random and points inward with respect to the surface $r=R$ (dashed circle).

keep pace with the algal cell. Inserting numbers, $v_{\mathrm{alg}} \approx 40 \mu \mathrm{m} \mathrm{s}^{-1}$ and $v_{\mathrm{bac}} \approx$ $200 \mu \mathrm{m} \mathrm{s}^{-1}$, so $\theta \approx 40 / 200=0.2$ radians $\approx 11^{\circ}$. While this scaling argument focusses on the effect of vorticity, the simulation results in section 3 show that advection by the algal cell's velocity field and rotation by its strain rate field are also important.

\subsection{Simulation method}

It is common practice to non-dimensionalise the quantities in a problem before performing simulations. In the problem considered here, there are six dimensional quantities of interest (five parameters plus time): algal cell radius $a$, algal cell swimming speed $v_{\text {alg }}$, bacterial cell swimming speed $v_{\text {bac }}$, reversal distance $R$, bacterial cell rotational diffusivity $D_{\mathrm{R}}$, and time $t$. In principle, by non-dimensionalising with respect to, say, the algal cell radius and the algal cell speed, one could reduce the number of quantities to just four. In practice, however, this is impractical, because then it is difficult to ascertain the effect of altering a single dimensional parameter. For this reason we shall define a non-dimensional time $t^{*}=t v_{\text {alg }} / a$, but leave other quantities dimensional. One unit of $t^{*}$ then corresponds to the time it takes the algal cell to swim a distance equal to its own radius.

For each simulation, the initial position of the model bacterial cell is chosen from a uniform, random distribution on the surface $r=R$, where $r$ denotes distance from the centre of the algal cell. The initial swimming direction of the bacterial cell is chosen from a uniform, random distribution on the hemisphere of unit vectors that point inward with respect to the surface $r=R$. An example initial condition is shown in figure 6. The algal cell position and the bacterial cell position and orientation are then evolved in time, using a simple Euler timestep scheme, according to the rules outlined in section 2.2. The time-step $\Delta t$ is chosen to be $\Delta t=0.005 a / v_{\text {alg. }}$. (Simulations were repeated using smaller time- 


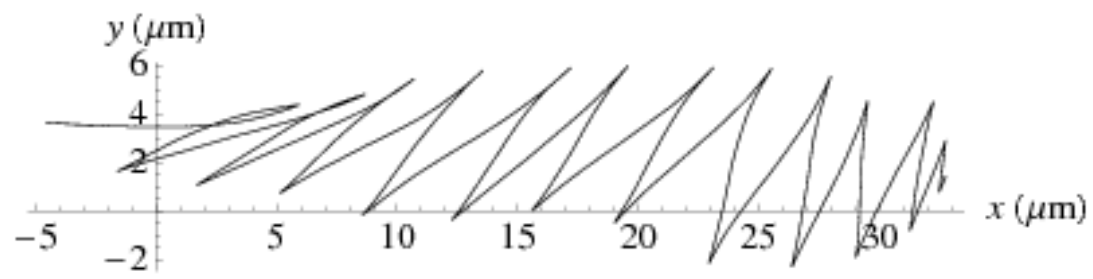

Figure 7: Example simulation trajectory of the model bacterial cell, projected onto a plane. The algal cell swims along the $x$-axis.

steps to check convergence of results.) Brownian rotation is simulated by giving the bacterial cell a new swimming direction at each time-step, chosen from an axisymmetric distribution about the old direction, such that the new direction makes an angle of $2 \sqrt{D_{\mathrm{R}} \Delta t}$ with the old direction 4. The bacterial cell is considered to be tracking the algal cell successfully for so long as it remains within the surface $r=R$, and is considered to have lost track of the algal cell if it strays outside this surface. Each simulation is terminated at either a pre-determined time, or when the bacterial cell loses track of the algal cell, whichever comes sooner.

Unless otherwise stated, the default parameter values used in the simulations are: algal cell radius $a=3 \mu \mathrm{m}$, reversal distance $R=6 \mu \mathrm{m}$, algal cell swimming speed $v_{\text {alg }}=40 \mu \mathrm{m} \mathrm{s}^{-1}$, bacterial cell swimming speed $v_{\text {bac }}=200 \mu \mathrm{m} \mathrm{s}^{-1}$, rotational diffusivity of bacterial cell $D_{\mathrm{R}}=0.062 \mu \mathrm{m}^{2} \mathrm{~s}^{-1}$, and bacterial cell slenderness ratio $\eta=10$. For each set of parameters, 500 simulations are run. We then calculate the 'empirical survival function' $S\left(t^{*}\right)$, defined as the fraction of simulations in which the bacterial cell is still tracking the algal cell at dimensionless time $t^{*}$. The standard error $S_{E}\left(t^{*}\right)$ of $S\left(t^{*}\right)$ is estimated by

$$
S_{E}\left(t^{*}\right)=\sqrt{S\left(t^{*}\right)\left[1-S\left(t^{*}\right)\right] / N}
$$

where $\mathrm{N}$ is the number of simulations 19 .

\section{Results}

We shall first present data for the default set of parameter values listed at the end of the previous section, and later look at the effect of altering the parameter values. Figure 7 shows an example trajectory of the model bacterial cell, projected onto a plane, for a simulation run with the default parameter values. The trajectory bears similarities to the experimental results shown in figure 1, especially in that the primary motion of the bacterial cell is almost at right angles to the motion of the algal cell, so that the bacterial cell's motion is a zigzag. This zigzag motion is not a once-off but occurs in most of the simulations, as revealed by the probability density function for the angle between the bacterial and algal swimming directions (figure 8). For the same data set, figure 10 shows the probability density of a bacterium being located at a polar angle $\theta$ in a coordinate system centred on the algal cell, where $\theta$ is measured from the algal cell's swimming direction. It shows that tracking most often occurs with the bacterial cell behind the algal cell rather than in front. 


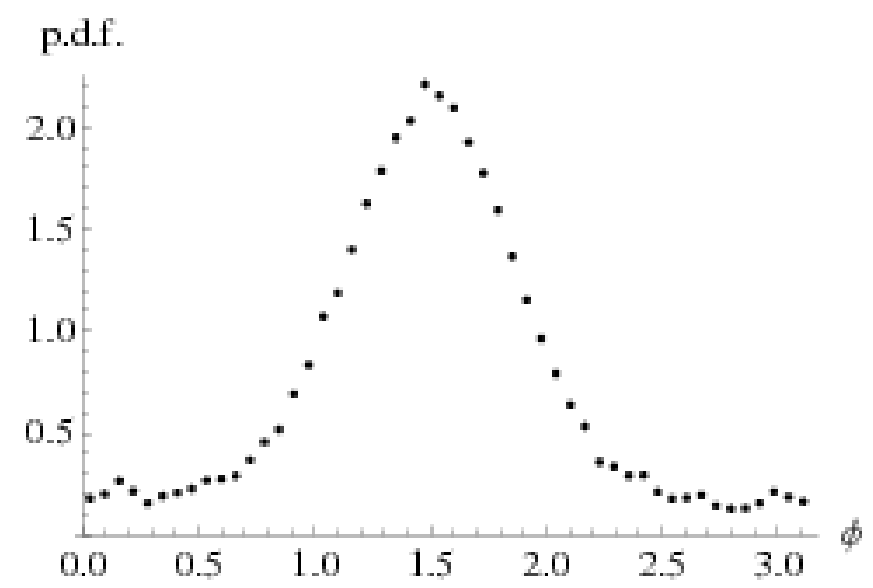

Figure 8: Approximate probability density function (p.d.f.) for the angle $\phi$ between the bacterial cell's swimming direction $\boldsymbol{p}$ and the algal cell's swimming direction, for a set of 500 simulations with the default parameter values. The p.d.f is normalised so that in the case of isotropically distributed $\boldsymbol{p}$ it would be equal to unity for all $\phi$. Note the peak near $\phi=\pi / 2$.

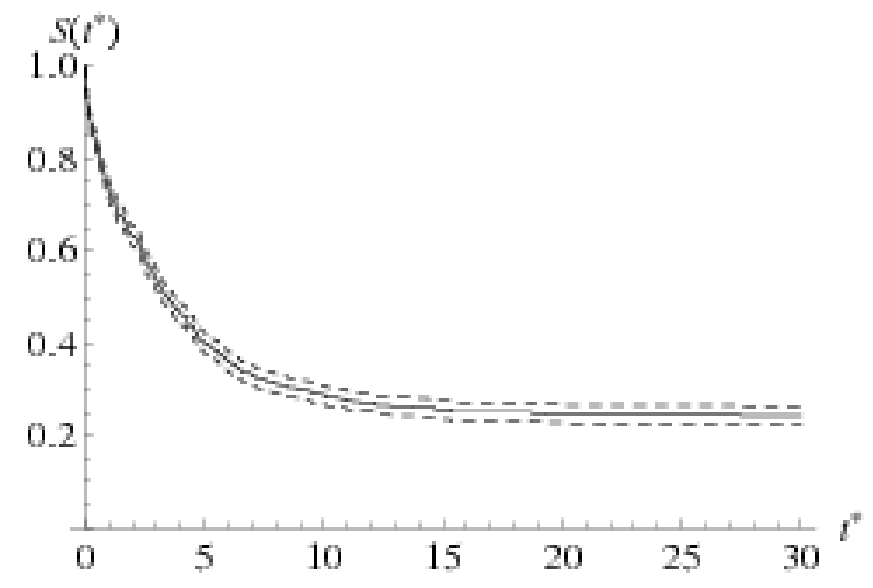

Figure 9: Solid line shows survival function $S\left(t^{*}\right)$, defined as the fraction of simulations in which duration of bacterial tracking is at least $t^{*}$, for a set of 500 simulations with the default parameter values. Dashed lines show error bounds $S\left(t^{*}\right) \pm S_{E}\left(t^{*}\right)$. 


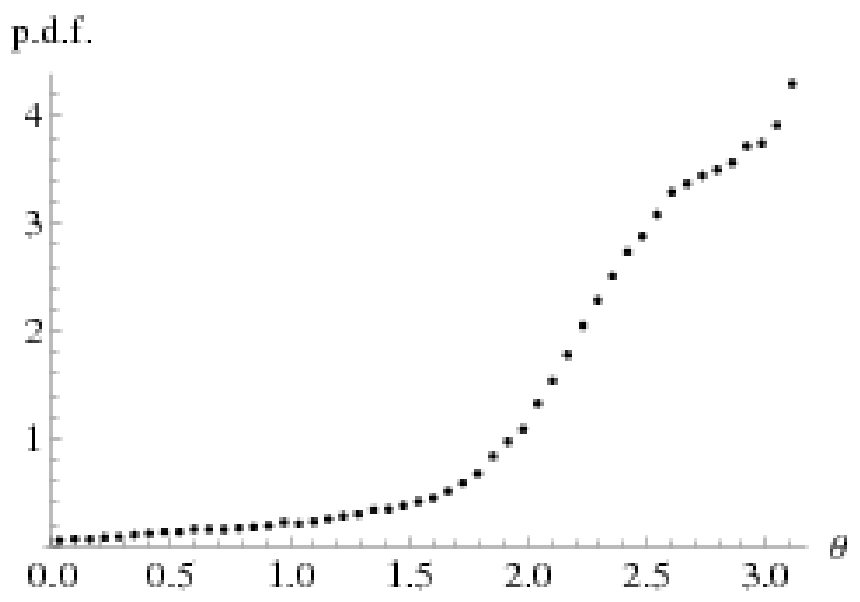

Figure 10: Approximate probability density function (p.d.f.) for the polar angle $\theta$ describing the position of the bacterial cell relative to the algal cell, for a set of 500 simulations with the default parameter values. The p.d.f. is normalised so that in the case of an isotropic distribution it would be equal to unity for all $\theta$.

Many of the tracking durations seen in the simulations are at least as long as those seen in experiments. Figure 9 shows the survival function and its standard error for 500 simulations, using the default parameter values. By way of comparison, the $P$. haloplanktis and $S$. putrefaciens bacteria in the experiments of [3] tracked on average for $0.7 \mathrm{~s}$ and $1.8 \mathrm{~s}$ respectively, corresponding to dimensionless tracking durations of $t^{*} \approx 9$ and $t^{*} \approx 23$. Note that the survival function appears to reach a steady value of $S \approx 0.25$, indicating that in a significant fraction of the simulations the bacterial cell is able to track the algal cell indefinitely. Extending the simulation duration up to $t^{*}=100$ reveals no further decay in $S$. Closer examination of the data reveals that in all cases where the tracking extends beyond $t^{*}=30$, the bacterial cell regularly collides with the algal cell, so re-orientations due to collisions play an important role.

The scaling argument presented in section 2.3 suggests that tracking can occur regardless of the precise value of the algal cell's swimming speed $v_{\text {alg. }}$. If the algal cell swims faster, for instance, then its vorticity field is proportionally stronger, and the consequent bacterial cell steering is more pronounced. In support of this, the simulation results show that the survival function is fairly insensitive to $v_{\text {alg }}$ over a large range $\left(20 \mu \mathrm{m} \mathrm{s}^{-1} \leq v_{\text {alg }} \leq 200 \mu \mathrm{m} \mathrm{s}^{-1}\right.$, figure 11), when tracking duration is measured by the dimensionless time $t^{*}$. Since one unit of $t^{*}$ corresponds to the algal cell swimming a distance equal to its own radius, the typical distance over which tracking occurs is independent of $v_{\text {alg }}$, but the absolute duration of tracking varies inversely with $v_{\text {alg. The scaling }}$ argument in section 2.3 also suggests that tracking does not depend on the precise value of the bacterial swimming speed $v_{\mathrm{bac}}$, and indeed the survival function was found to have no statistically significant dependence on $v_{\mathrm{bac}}$ over the range $40 \mu \mathrm{m} \mathrm{s}^{-1} \leq v_{\mathrm{bac}} \leq 400 \mu \mathrm{m} \mathrm{s}^{-1}$ (data not shown), while holding all other parameters at their default values.

Where the scaling argument fails is in its prediction that tracking is not 


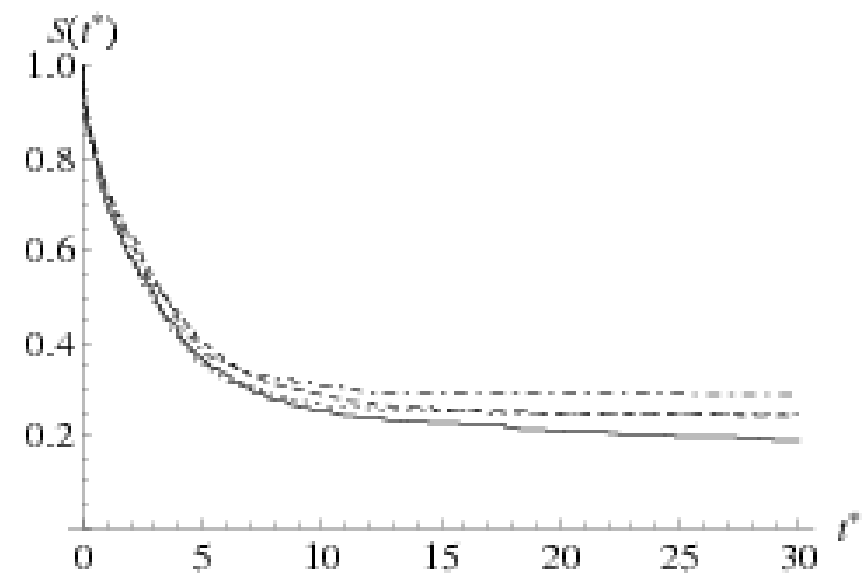

Figure 11: Survival function $S\left(t^{*}\right)$ for $v_{\text {alg }}=20 \mu \mathrm{m} \mathrm{s}^{-1}$ (solid), $v_{\text {alg }}=40 \mu \mathrm{m} \mathrm{s}^{-1}$ (dashed), $v_{\text {alg }}=80 \mu \mathrm{m} \mathrm{s}^{-1}$ (dotted), and $v_{\text {alg }}=200 \mu \mathrm{m} \mathrm{s}^{-1}$ (dot-dashed). Standard errors (not shown) are of the same magnitude as those in figure 9 .

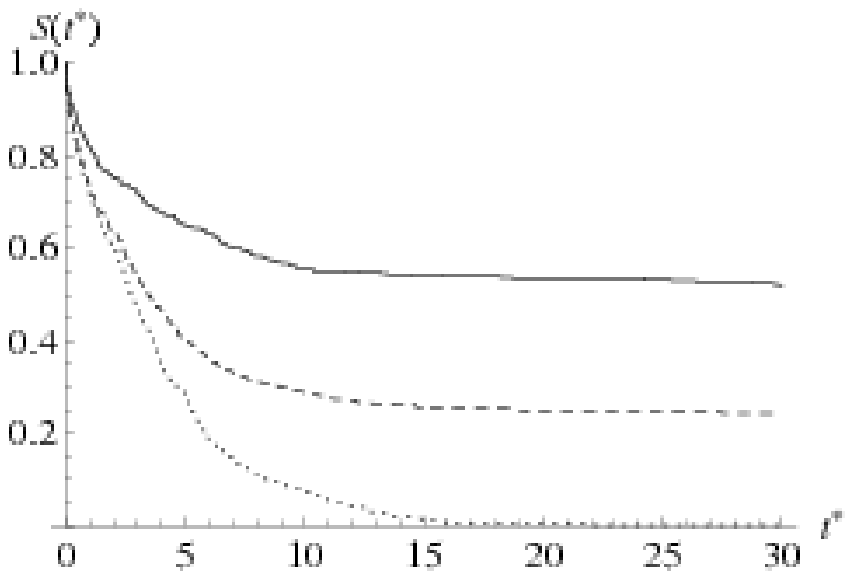

Figure 12: Survival function $S\left(t^{*}\right)$ for reversal distance $R=4 \mu \mathrm{m}$ (solid), $R=6 \mu \mathrm{m}$ (dashed), and $R=9 \mu \mathrm{m}$ (dotted). Standard errors (not shown) are of the same magnitude as those in figure 9 . 


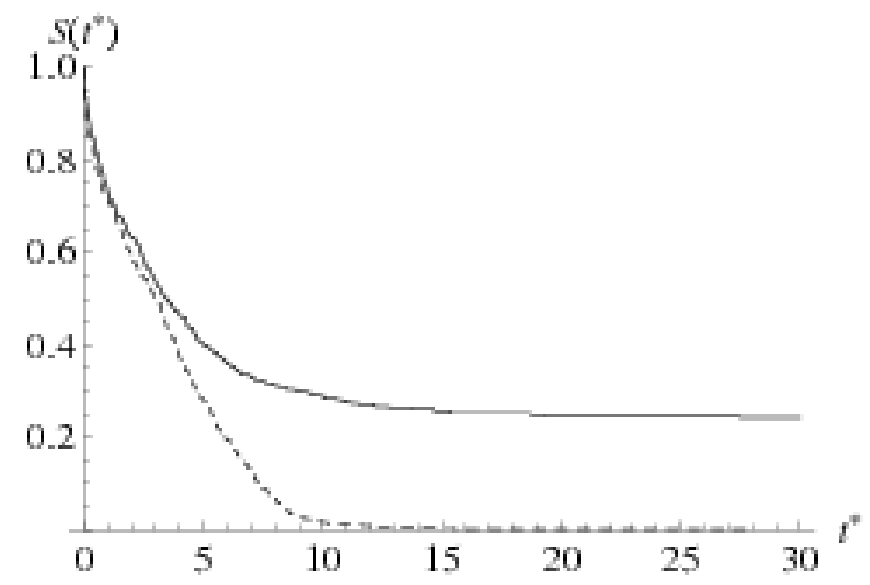

Figure 13: Survival function $S\left(t^{*}\right)$ for default set of parameters (solid line), and for the same set of parameters but with rotation by vorticity and strain rate field turned off (dashed line). Standard errors (not shown) are of the same magnitude as those in figure 9 .

sensitive to the reversal distance $R$. As shown in figure 12, there is a strong inverse relation between $R$ and tracking duration. The main reason why the scaling argument fails is because it does not account for advection of the bacterial cell by the algal cell's velocity field, which turns out to be important. Taking a time-average of (11), the time-averaged bacterial cell velocity $\langle\dot{\boldsymbol{x}}\rangle$ has a swimming contribution $v_{\text {bac }}\langle\boldsymbol{p}\rangle$ and an advective contribution $\langle\boldsymbol{u}\rangle$, where angle brackets denote a time-average. For simulations with the default set of parameters, swimming contributes $\approx 30 \%$ and advection contributes $\approx 70 \%$ to the time-averaged bacterial cell velocity. For larger $R$, the advection term is smaller, and the bacterial cell has less chance of keeping up with the algal cell.

Given that advection contributes the dominant fraction of the time-averaged bacterial cell velocity, one might ask how important swimming and steering really are. One way to test this is to artificially remove the deterministic terms involving $\boldsymbol{\omega}$ and $\boldsymbol{E}$ on the right hand size of (2), so that there is no 'hydrodynamic steering'. The swimming direction $\boldsymbol{p}$ then evolves only due to collisions, reversals, and Brownian rotation. As shown in figure 13, bacterial tracking is markedly impaired when hydrodynamic steering is turned off. Thus, even though advection is the dominant contribution to the net bacterial motion, on its own it is not sufficient to enable longer duration tracking. This concurs with the observations of [3], who, using latex beads as passive substitutes for bacteria, found that there was no tracking or entrainment of beads by any algae.

The rate of change of bacterial swimming direction induced by the algal cell's strain-rate field $\boldsymbol{E}$ depends on the elongation $\eta$ of the bacterial cell, through (2) and (3). A spherical cell is not rotated at all by the strain-rate field, and an elongated cell is rotated so as to align with the direction of maximum stretching. Since the direction of maximum stretching points approximately toward or away from the algal cell (figure (1), one expects a more elongated bacterial cell to have a superior tracking performance. Figure 14 shows that this is indeed the case. For large $\eta$, the precise value is not important; survival curves for different $\eta$ 


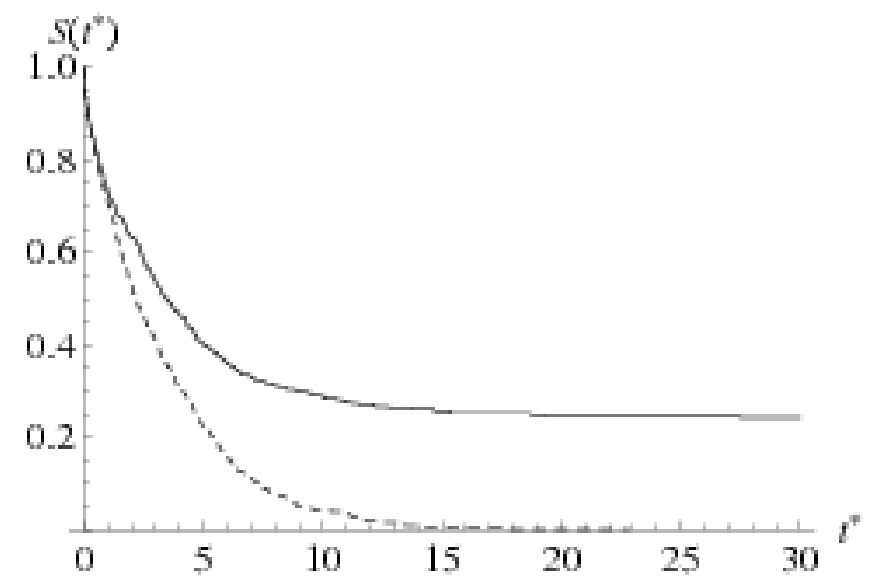

Figure 14: Survival function $S\left(t^{*}\right)$ for an elongated bacterial cell $(\eta=10$, solid line), and for an spherical bacterial cell ( $\eta=1$, dashed line). Standard errors (not shown) are of the same magnitude as those in figure 9.

beyond $\eta \approx 5$ lie almost on top of one another (data not shown).

As explained in section 2.2 the rotational diffusion coefficient $D_{\mathrm{R}}$ for the bacterial cell is difficult to estimate, because it may be due to 'wobbly swimming' rather than true, thermal, Brownian rotation. Figure 15 shows that in any case there is only a weak dependence on $D_{\mathrm{R}}$. A large value of $D_{\mathrm{R}}$ does not significantly affect the survival function at early times $t^{*}<20$, but causes a slow decay in the survival function at longer times, so a bacterial cell cannot continue tracking indefinitely.

\section{Discussion}

The simulations presented in this paper suggest that the bacterial cell steering seen by Barbara and Mitchell [3] could be a passive hydrodynamic effect. The model bacterial cell in the present study does not actively steer, it simply swims forward and back, and through its interaction with the model algal cell's velocity, vorticity and strain rate fields (all three of which are important) it is able to track the model algal cell. The simulated bacterial cell trajectories have a similar zigzag shape to those seen in experiments, and some of the tracking durations seen in the simulations are at least as long as those seen in the experiments. Indeed some tracking events last indefinitely (for as long as the simulations are run), though in reality such long tracking events would be disrupted by effects such as unsteadiness in the algal cell's velocity field, changes in the algal cell's swimming direction, or noise in the bacterial cell's chemosensory system.

The simulations indicate that the reversal distance $R$ (the distance from the algal cell at which the bacterial cell reverses direction) has a very strong effect on tracking duration, with smaller $R$ enabling longer tracking duration. The reversal distance is an artificial construct of the model, since in reality the bacterial cell's chemotactic response is almost certainly more complicated than simply reversing direction whenever the chemoattractant concentration drops below a certain threshold. Even so, the inverse relation between $R$ and 


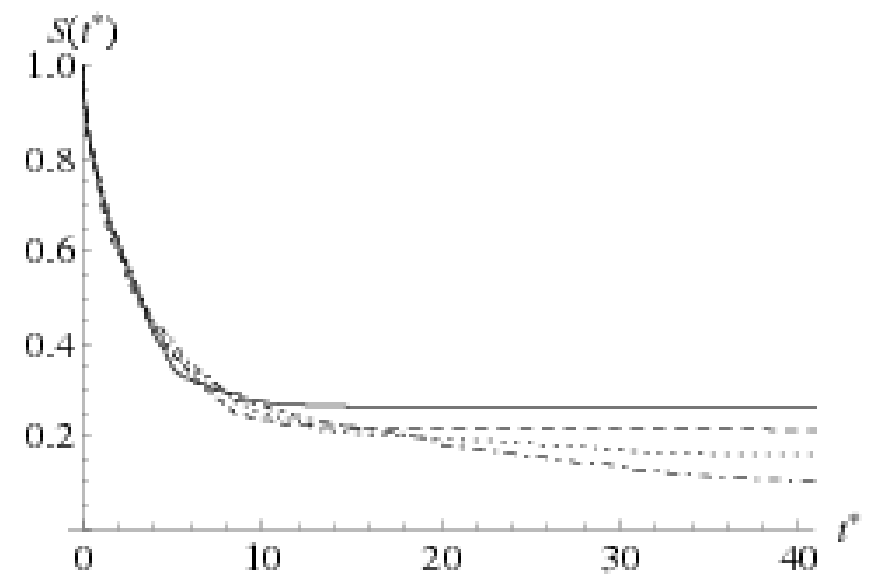

Figure 15: Survival function $S\left(t^{*}\right)$ for bacteria with rotational diffusion coefficients $D_{\mathrm{R}}$ of 0 radians $^{2} \mathrm{~s}^{-1}$ (solid), 0.062 radians $^{2} \mathrm{~s}^{-1}$ (dashed), 0.12 radians $^{2} \mathrm{~s}^{-1}$ (dotted), and 0.2 radians $^{2} \mathrm{~s}^{-1}$ (dot-dashed). Standard errors (not shown) are of the same magnitude as those in figure 9 .

tracking duration shows that the ability to respond rapidly to chemoattractant signals and reverse direction with high frequency is crucial in order for tracking to occur, and this may represent an evolutionary pressure on certain marine bacteria toward faster response times.

Another result of our simulations is that the dimensionless tracking duration (proportional to the number of body lengths the algal cell swims while being tracked) is largely independent of the algal and bacterial swimming speeds. This seems at odds with the experimental results, where [3] found that bacteria increase their swimming speed by roughly a factor of two when tracking (relative to not-tracking). If tracking occurs just as well at lower bacterial swimming speeds, why do the bacteria swim faster? First, the faster swimming speed increases diffusive flux, and hence nutrient acquisition, by about $30 \%$ 3. Second, it could also be that faster swimming speeds result in more robust tracking when the algal cell changes swimming direction, a complication not included in our model. Third, it could be that some bacteria in the culture were committed to anaerobic metabolism, and only the faster-swimming aerobically metabolising cells performed tracking (J. G. Mitchell, personal communication).

Perhaps the most serious limitation of the present model is that it treats the bacterial cell as being infinitesimally small, whereas in reality its length is comparable to the algal cell diameter. Thus, instead of being rotated by the vorticity and strain-rate fields at a point in space, in reality the bacterial cell 'samples' these fields over its length. In defense of the present model, we point to the work of [37], who performed a combined experimental and theoretical investigation of the re-orientation of elongated food particles (diatoms) in copepod feeding currents. The food particles in their experiments were of similar length to the copepods generating the currents, but the authors still found convincing agreement with theoretical calculations that treated the food particles as infinitesimal.

Given the importance of the algal cell's vorticity and strain rate fields in 
re-orienting the bacterial cell, it is natural to ask whether ambient, turbulent fields might disrupt tracking. However, the strength of the strain rate tensor due to turbulence varies from about $1.5 \mathrm{~s}^{-1}$ in the upper mixed layer of the ocean under strong wind forcing to $0.005 \mathrm{~s}^{-1}$ at the thermocline [23], and these magnitudes are small compared with the strain rate of $v_{\text {alg }} / R \approx 7 \mathrm{~s}^{-1}$ in the vicinity of the algal cell. Thus, turbulence is unlikely to disrupt tracking. It might, of course, play a role in how the bacterial cell finds the algal cell in the first place, which is not addressed in our model. Another consideration related to the strength of the algal cell's strain rate field is whether it might be strong enough to deform the bacterial cell's flagellum. An order of magnitude estimate using the bending stiffness reported by [7] for an E. coli flagellum suggests that deformation could be significant. This provides another potential mechanism for passive hydrodynamic steering, which has not yet been explored.

The idea that microorganisms may exploit vorticity and strain rate fields in their feeding behaviours is not new. In [30], it is suggested that certain freshwater Crustacea (cladocerans), which feed by intermittently swimming vertically upward and sinking down again, might enhance their prey capture rate through hydrodynamic effects. Their calculations show that bottom heavy motile algae are focussed into the wake of one of these sinking crustaceans by its vorticity field ('gyrotaxis'), whereupon the crustacean presumably consumes the algae when it swims up. In [34], a similar analysis is performed for the case of a bottom heavy flagellate focussed into the wake of a nonmotile, sinking algal cell. In the simulations of 23, elongated model bacteria using a run-and-reverse ('back-and-forth') motion are able to stay close to a nutrient source even at high ambient shear, because the strain rate field aligns the bacteria toward the nutrient patch.

While the simulations presented in this paper suggest that the apparent steering seen by 33 could be a passive hydrodynamic effect, the results do not rule out the possibility that the bacteria are actively steering as well. The fact that bacteria were able to follow 'ghost tracks' some distance behind algal cells (mentioned at the bottom of p82 of [3]) suggests that there may also be active steering, since vorticity and strain rate fields far behind the cell are probably not sufficient to rotate the cell. If $P$. haloplanktis and $S$. putrefaciens are indeed capable of steering, then they are not the first bacteria to be found to do so. Experiments indicate that another (unnamed) species of bacteria can sense an oxygen gradient over its body length and steer relative to the gradient in a continuous fashion [33], though it is quite different in shape from P. haloplanktis and $S$. putrefaciens. In another example, Rhodobacter sphaeroides has a similar shape to $P$. haloplanktis and $S$. putrefaciens, with a single flagellum, and is able to changes direction by altering the conformation of this helix [1], though there is no evidence of directed steering in this case.

Finally, we should note that it has not been our intention in this report to make light of the work of [3]. Their finding of tracking bacteria is interesting in its own right and nutrient turnover by tracking bacteria could play a key role in the food web especially if, as the authors suggest, a similar mechanism applies to the tracking of marine snow. Rather, our intention has been to elucidate the intriguing possibility that not only are the marine bacteria able to perform tracking, but that they do so by exploiting the velocity, vorticity, and strain-rate fields of their quarry. 


\section{Acknowledgements}

J. T. Locsei is supported by an Oliver Gatty Studentship from the University of Cambridge. The authors are grateful to J. G. Mitchell for helpful discussions.

\section{References}

[1] Judith P. Armitage, Thomas P. Pitta, Margot A. S. Vigeant, Helen L. Packer, and Roseanne M. Ford. Transformations in flagellar structure of Rhodobacter sphaeroides and possible relationship to changes in swimming speed. J. Bacteriol., 181(16):4825-4833, 1999.

[2] Farooq Azam. Microbial Control of Oceanic Carbon Flux: The Plot Thickens. Science, 280(5364):694-696, 1998.

[3] Greg M. Barbara and James G. Mitchell. Bacterial tracking of motile algae. FEMS Microbiol. Ecol., 44(1):79-87, 2003.

[4] H. C. Berg. Random Walks in Biology. Princeton University Press, 1983.

[5] Howard C. Berg and Douglas A. Brown. Chemotaxis in Escherichia coli analysed by three-dimensional tracking. Nature, 239:500-504, October 1972 .

[6] James D. Bowen, Keith D. Stolzenbach, and Sallie W. Chisholm. Simulating bacterial clustering around phytoplankton cells in a turbulent ocean. Limnol. Oceanogr., 38(1):36-51, 1993.

[7] Nicholas C. Darnton, Linda Turner, Svetlana Rojevsky, and Howard C. Berg. On torque and tumbling in swimming Escherichia coli. J. Bacteriol., 189(5):1756-1764, 2007.

[8] Willow R. DiLuzio, Linda Turner, Michael Mayer, Piotr Garstecki, Douglas B. Weibel, Howard C. Berg, and George M. Whitesides. Escherichia coli swim on the right-hand side. Nature, 435:1271-1274, 2005.

[9] Tom Fenchel. Eppur si muove: many water column bacteria are motile. Aquat. Microb. Ecol., 24:197-201, 2001.

[10] G Gauthier M Gauthier and R Christen. Phylogenetic analysis of the genera Alteromonas, Shewanella, and Moritella using genes coding for smallsubunit rRNA sequences and division of the genus Alteromonas into two genera, Alteromonas (emended) and Pseudoalteromonas gen. nov., and proposal of twelve new species combinations. Int. J. Syst. Bacteriol., 45:755$761,1995$.

[11] Stuart F Goldstein. Flagellar beat patterns in algae. In Michael Melkonian, editor, Algal Cell Motility, number 3 in Current Phycology, chapter 4. Chapman and Hall, 1st edition, 1992.

[12] Hans-Peter Grossart, Lasse Riemann, and Farooq Azam. Bacterial motility in the sea and its ecological implications. Aquat. Microb. Ecol., 25:247-258, September 2001. 
[13] Juan P. Hernandez-Ortiz, Christopher G. Stoltz, and Michael D. Graham. Transport and collective dynamics in suspensions of confined swimming particles. Phys. Rev. Lett., 95(20):204501, 2005.

[14] H M Holt, B Gahrn-Hansen, and B Bruun. Shewanella algae and Shewanella putrefaciens: clinical and microbiological characteristics. Clin. Microbiol. Inf., 11(5):347-352, 2005.

[15] George A Jackson. Simulating chemosensory responses of marine microorganisms. Limnol. Oceanogr., 32(6):1253-1266, 1987.

[16] George A Jackson. Simulation of bacterial attraction and adhesion to falling particles in an aquatic environment. Limnol. Oceanogr., 34(3):514-530, 1989.

[17] S. Kim and S. J. Karrila. Microhydrodynamics: Principles and Selected Applications. Butterworth Heinemann, 1992.

[18] Thomas Kiorboe, Hans-Peter Grossart, Helle Ploug, and Kam Tang. Mechanisms and rates of bacterial colonization of sinking aggregates. Appl. Environ. Microbiol., 68(8):3996-4006, 2002.

[19] Jerald F Lawless. Statistical models and methods for lifetime data. Wiley series in probability and statistics. John Wiley \& Sons, 2003.

[20] L G Leal and E J Hinch. The rheology of a suspension of nearly spherical particles subject to Brownian rotations. J. Fluid Mech., 55:745-65, 1972.

[21] $\mathrm{J} \mathrm{T}$ Locsei and $\mathrm{T} \mathrm{J}$ Pedley. Run and tumble chemotaxis in a shear flow: the effect of temporal comparisons and other complications. arXiv:0804.2352v1, 2008.

[22] Janos Tobias Locsei. Persistence of direction increases the drift velocity of run and tumble chemotaxis. J. Math. Biol., 55(1):41-60, 2007.

[23] Rolph H. Luchsinger, Birger Bergerson, and James G. Mitchell. Bacterial swimming strategies and turbulence. Biophys. J., 77:2377-2386, November 1999.

[24] Y Magariyama, S Sugiyama, K Muramoto, Y Maekawa, I Kawagishi, Y Imae, and S Kudo. Very fast flagellar rotation. Nature, 371(6500):752, 1994.

[25] T H Mague, E. Friberg, D J Hughes, and I. Morris. Extracellular release of carbon by marine phytoplankton; a physiological approach. Limnol. Oceanogr., 25(2):262-279, 1980.

[26] J G Mitchell, M Martinez-Alonso, J Lalucat, I Esteve, and S Brown. Velocity changes, long runs, and reversals in the Chromatium minus swimming response. J. Bacteriol., 173(3):997-1003, 1991.

[27] J G Mitchell, L Pearson, and S Dillon. Clustering of marine bacteria in seawater enrichments. Appl. Environ. Microbiol., 62(10):3716-3521, 1996. 
[28] James G. Mitchell and Kazuhiro Kogure. Bacterial motility: links to the environment and a driving force for microbial physics. FEMS Microbiol. Ecol., 55(1):3-16, 2006.

[29] James G Mitchell, Lynette Pearson, Simon Dillon, and Katerina Kantalis. Natural assemblages of marine bacteria exhibiting high-speed motility and large accelerations. Appl. Environ. Microbiol., 61(12):4436-4440, 1995.

[30] T. J. Pedley and J. O. Kessler. The orientation of spheroidal microorganisms swimming in a flow field. Proc. R. Soc. Lond. B, 231(1262):47-70, 1987.

[31] E M Purcell. Life at low Reynolds number. Am. J. Phys., 45:3-11, 1977.

[32] Jeffrey E. Segall, Steven M. Block, and Howard C. Berg. Temporal comparisons in bacterial chemotaxis. Proc. Nat. Acad. Sci. USA, 83(23):8987-8991, December 1986

[33] Roland Thar and Michael Kühl. Bacteria are not too small for spatial sensing of chemical gradients: An experimental evidence. Proc. Nat. Acad. Sci. USA, 100(10):5748-5753, 2003.

[34] Uwe Timm and Akira Okubo. Gyrotaxis: interaction between algae and flagellates. Bull. Math. Biol., 57(5):631-650, 1995.

[35] Linda Turner, William S. Ryu, and Howard C. Berg. Real-time imaging of fluorescent flagellar filaments. J. Bacteriol., 182(10):2793-2801, 2000.

[36] Kasthuri Venkateswaran, Duane P. Moser, Michael E. Dollhopf, Douglas P. Lies, Daad A. Saffarini, Barbara J. MacGregor, David B. Ringelberg, David C. White, Miyuki Nishijima, Hiroshi Sano, Jutta Burghardt, Erko Stackebrandt, , and Kenneth H. Nealson. Polyphasic taxonomy of the genus Shewanella and description of Shewanella oneidensis sp. nov. Int. J. Syst. Bacteriol., 49:705-724, 1999.

[37] Andre W Visser and Per R Jonsson. On the reorientation of non-spherical prey particles in a feeding current. J. Plankton Res., 22(4):761-777, 2000. 